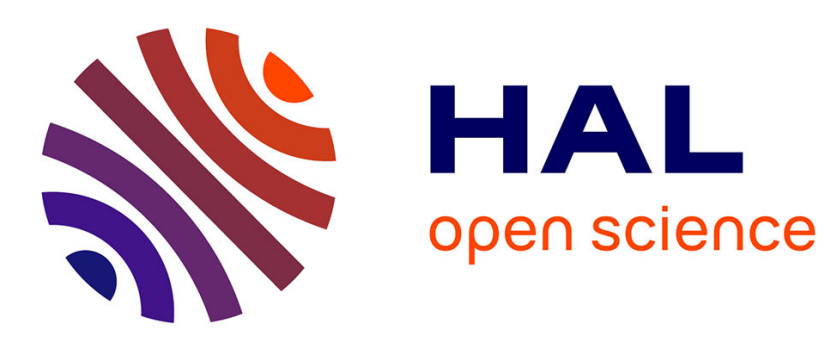

\title{
Mechanical Spectroscopy of Side Chain Liquid Crystalline Polymers
}

S. Etienne, L. David, P. Sixou, C. Laye

\section{To cite this version:}

S. Etienne, L. David, P. Sixou, C. Laye. Mechanical Spectroscopy of Side Chain Liquid Crystalline Polymers. Journal de Physique IV Proceedings, 1996, 06 (C8), pp.C8-571-C8-574. 10.1051/jp4:19968123 . jpa-00254554

\section{HAL Id: jpa-00254554 https://hal.science/jpa-00254554}

Submitted on 1 Jan 1996

HAL is a multi-disciplinary open access archive for the deposit and dissemination of scientific research documents, whether they are published or not. The documents may come from teaching and research institutions in France or abroad, or from public or private research centers.
L'archive ouverte pluridisciplinaire HAL, est destinée au dépôt et à la diffusion de documents scientifiques de niveau recherche, publiés ou non, émanant des établissements d'enseignement et de recherche français ou étrangers, des laboratoires publics ou privés. 


\title{
Mechanical Spectroscopy of Side Chain Liquid Crystalline Polymers
}

\author{
S. Etienne, L. David*, P. Sixou** and C. Laye** \\ Laboratoire de Métallurgie Physique et Science des Matériaux, UA 155 du CNRS, Ecole des Mines, Parc \\ de Saurupt, 54042 Nancy cedex, France \\ * Groupe d'Etude de Métallurgie Physique et Physique des Matériaux, Institut National des Sciences \\ Appliquées de Lyon, 20 avenue A. Einstein, 69621 Villeurbanne cedex, France \\ ** Laboratoire de Physique de la Matière Condensée, UA 190 du CNRS, Université de Nice Sophia- \\ Antipolis, Parc Valrose, 06108 Nice cedex 2, France
}

\begin{abstract}
Summary: we report a low frequency mechanical relaxation study of molecular motions near and below glass transition in side chain liquid crystal polymers (SCLCP) in the cholesteric state. The SCLCP was a blend of crosslinked and uncrosslinked materials. Different compositions were studied. Thesc materials exhibit low temperature relaxation processes and a main relaxation connected to the glass transition. Localised motions are responsible for the low temperature processes while the main relaxation exlibits cooperative features and is sensitive to the amount of crosslinked fraction. The relaxation time in the glass transition range when plotted against normalised reciprocal temperature shows that the molecular motions display a fragile character in agreement with the highly cooperative feature and strong physical aging effects. These observations are discussed in the frame of correlated motions involved in the liquid glass transition.
\end{abstract}

\section{INTRODUCTION}

In a previous work [1] we reported a first low frequency mechanical spectroscopy study of molecular motions in the liquid glass transition range in side chain liquid crystal polymers (SCLCP) with a short alkyl spacer length in the cholesteric state. This work is now extended in two ways: i) in order to improve the sensitivity of measurements, free standing thin specimens are used thus allowing investigation down to low temperature making it possible the study of sub $\mathrm{Tg}$ relaxations ii) different compositions with different amount of crosslinking were studied. Crosslinking is expected to affect the dynamic behavior particularly in the liquid glass transition range.

\section{MATERIALS AND METHODS}

\subsection{Materials and preparation of specimens}

Mixtures with different amounts of liquid crystalline polysiloxane and liquid crystalline poly(siloxane methacrylate), hereafter called S and SM, respectively, were prepared. The polysiloxane is a copolymer with two types of mesogenic groups attached to the main chain via short spacers: biphenyl groups (nematic) and cholesteric groups (chiral) [1]. The poly(siloxane methacrylate) fraction can be crosslinked by UV radiation exposure, in presence of a photoinitiator (irgacure 907, Ciba-Ceigy) as catalyst. The compositions are all indicated as weight fractions. The mixtures are heated up to $373 \mathrm{~K}$ to form platelets $0.3 \mathrm{~mm}$ thick. Then the material is subjected to UV radiation for $30 \mathrm{~min}$. Specimens are cut from platelets in suited shape $0.3 \times 2 \times 8 \mathrm{~mm}^{3}$ for mechanical spectroscopy experiments.

\subsection{Methods}

The dynamic mechanical behavior was assessed by means of a low frequency spectrometer working in torsion and described elsewhere [2]. It was possible to measure the dynamic shear modulus in isothermal 
(frequency scanning) or isochronal (temperature scanning) conditions in the temperature/frequency ranges $90 \mathrm{~K}-573 \mathrm{~K} / 10^{-5}-5 \mathrm{~Hz}$. The shear strain did not exceed $10^{-5}$.

\section{RESULTS}

Specimens of different compositions $(1-x) \mathrm{S}, x \mathrm{SM}$ with $x$ varying from zero to one were studied in order to investigate the effect of crosslinking rate. Isochronal spectra clearly display for each specimen low temperature relaxation effects $\beta 1$ and $\beta 2$ near $119 \mathrm{~K}$ and $195 \mathrm{~K}$ (frequency $1 \mathrm{~Hz}$ ), respectively (figure 1).

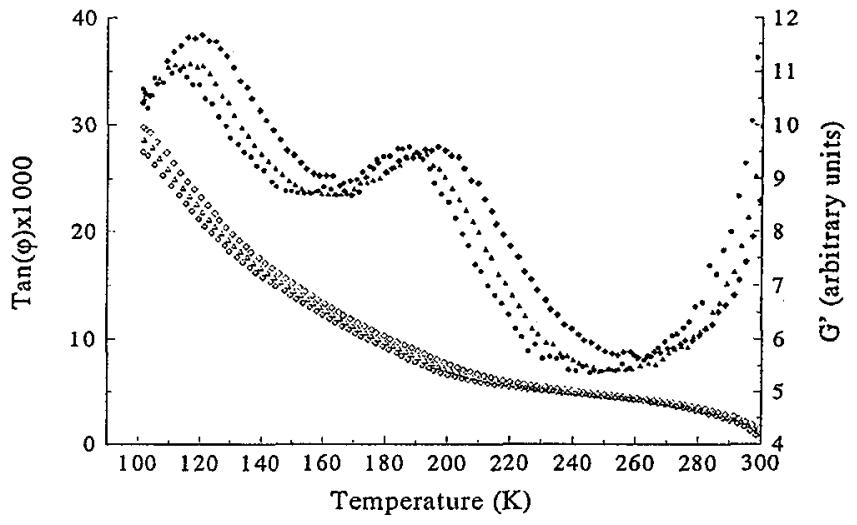

Figure 1: low temperature relaxation processes $\beta_{1}$ and $\beta_{2}$ observed at 3 frequencies for material with $x=0.25(0.2 \%$ catalyst). $\bullet: 1 \mathrm{~Hz}, \mathbf{\Lambda}: 0.3 \mathrm{~Hz}$ and $0: 0.09 \mathrm{~Hz}$. Empty symbols are relative to modulus $G^{\prime}$.
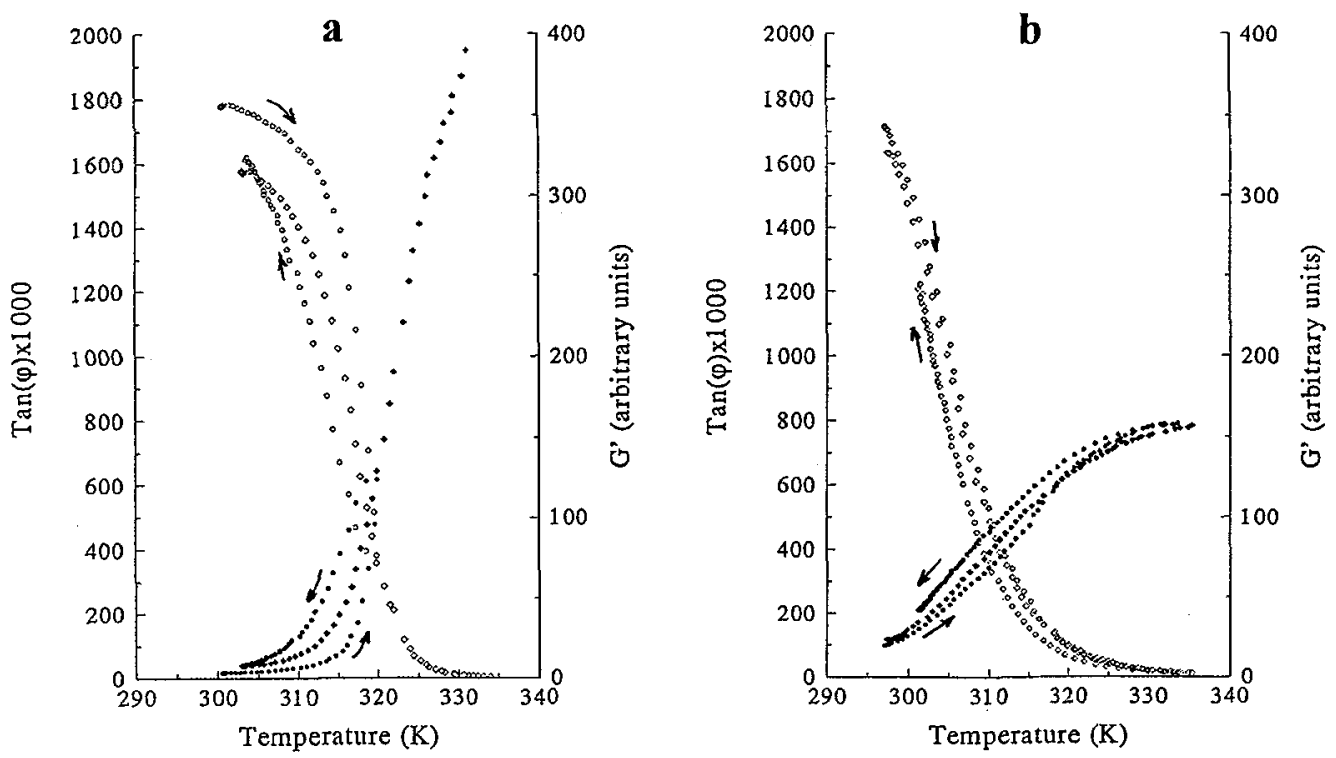

Figure2: relaxation process observed near the glass transition range. Only isochronal curves $1 \mathrm{~Hz}$ are drawn. The effect of physical aging is obvious by comparison of first heating $(\Theta)$, subsequent cooling $(\Theta)$ and second heating $(\bullet)$. a: $x=0$, b: $x=1$ ( $2 \%$ catalyst). Empty symbols are relative to modulus. 
The spectra taken at different frequencies during a temperature scan allow to deduce the apparent activation energy, $0.31 \mathrm{eV}$ and $0.50 \mathrm{eV}$ for $\beta 1$ and $\beta 2$, respectively. Above $290 \mathrm{~K}$, a strong relaxation effect associated with a drastic decrease of modulus over several orders of magnitude can be observed. Results for compositions $x=0$ and $x=1$ are displayed in figure 2 where the effect of physical aging is apparent.

Near the glass transition the mechanical relaxation time $\tau_{\mathrm{mn}}$ is extracted from measurements of the dynamic modulus as a function of frequency in isothermal conditions according to $\omega \tau_{\mathrm{mm}}=1$ when the imaginary component $G^{\prime \prime}$ is maximum. (figure 3). Because of physical aging effects, two regimes are to be distinguished: isoconfigurational $(T<T \mathrm{~g})$ and liquid equilibrium state $(T>T \mathrm{~g})$. According to figure 3 the liquid glass transition temperature is close to $291 \mathrm{~K}$ for a relaxation time of the order of $10^{5} \mathrm{~s}$ (this temperature is determined from extrapolations relative to isostructural and equilibrium state curves: broken lines in figure 3). Strong departure from a simple relaxation process is evident from the distorded shape of the Cole Cole diagram (figure 4), which agrees with the half width of the $\tan \varphi$ master curve (not shown here). The distorsion is characterised by the low values of the parameters $b$ and $b^{\prime}$ which are caiculated from $\lim _{w \rightarrow \infty}\left(\partial G^{\prime \prime} / \partial G^{\prime}\right)=-\tan b \pi / 2$ and $\lim _{w \rightarrow 0}\left(\partial G^{\prime \prime} / \partial G^{\prime}\right)=\tan b^{\prime} \pi / 2$. The parameter $b^{\prime}$ decreases when the crosslinking ratio increases: it varies from 0.758 down to 0.41 when $x$ varies from 0 up to 1 while in the same time $b$ remains nearly constant lying in the range 0.16 to 0.22 .

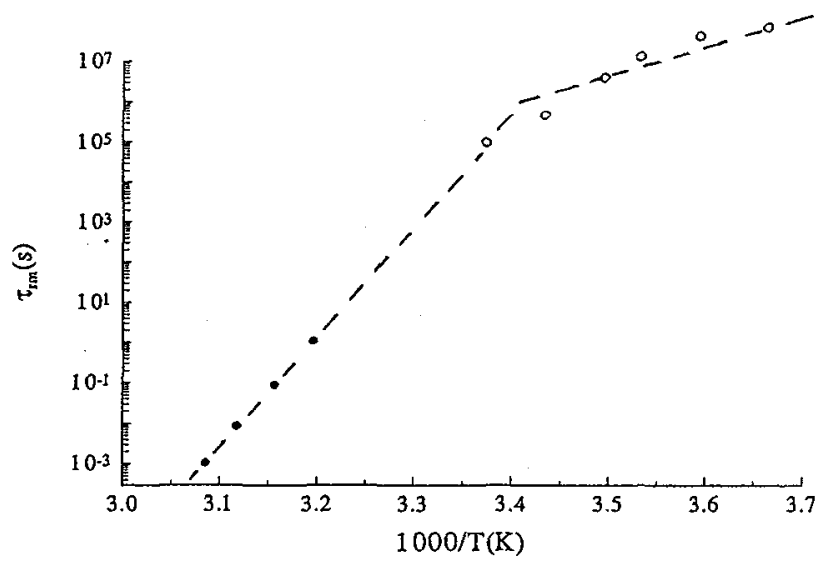

Figure 3: variation of the mechanical relaxation time as a function of temperature $(x=0.25,2 \%$ catalyst). Isoconfigurational empty circles and equilibrium state dark circles regimes are clearly distinguished.

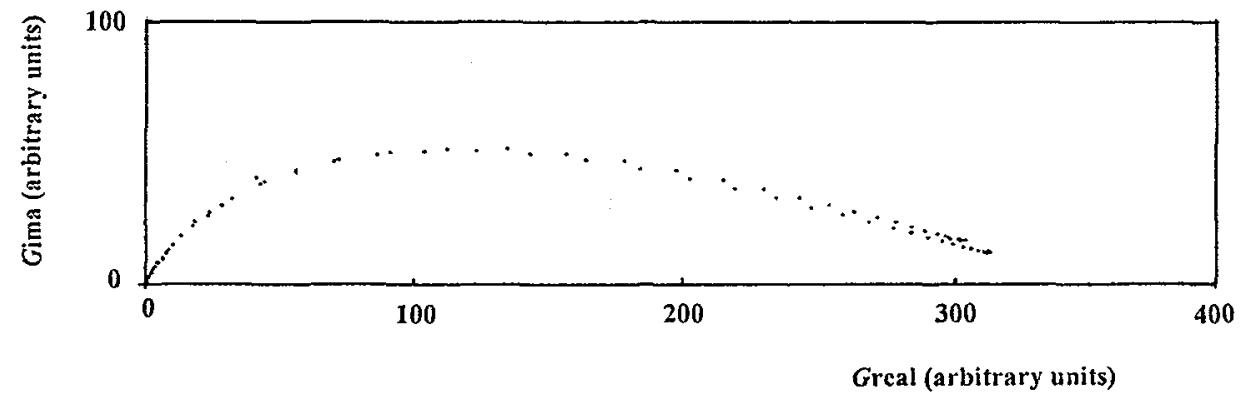

Figure 4: Colc Colc diagram $(x=0)$. 


\section{DISCUSSION AND CONCLUSION}

The low temperature $\beta 1$ and $\beta 2$ relaxation processes in SCLCP are reported for the first time in this paper. They can be regarded as simple relaxations according to their activation energy and frequency factor. Furthermore, they are present in the whole composition range invesgated in this work. As a result, they are assigned to rotational motions of mesogenic groups around their long axes.

The analysis of the main relaxation process is more complex since motions of mesogenic groups which couple with that of the main chain are involved. The complex modulus in the glass transition range can be written as: $G^{*}(j \omega)=G_{\mathrm{r}}+\left(G_{\mathrm{u}}-G_{\mathrm{r}}\right)\left[1+\left(j \omega \tau_{\mathrm{nn}}\right)^{-b}+\left(j \omega \tau_{\mathrm{rm}}\right)^{-b^{b}}\right]^{-1} . G_{\mathrm{u}}$ and $G_{\mathrm{r}}$ are the unrelaxed and relaxed modulus, respectively. The parameter $b$ is equivalent to the exponent of the stretched exponential relaxation function in the time domain. Its value is deduced from the high frequency slope limit of the Cole Cole plot. The low value of $b$ indicates highly cooperative or correlated motions, which is in good agreement with the fragile character of the liquid that manifests in figure 3 and strong aging effects shown in figure 2. Low values of $b$ where also observed in main chain LCP [3]. The fragile [4] behavior is consistent with the drastic sensitivity of the relaxation time near $T \mathrm{~g}$ in the liquid state (apparent activation energy about $7.7 \mathrm{eV}$ ) compared to that observed in the isoconfigurational state in the same temperature range (apparent activation energy about $1.5 \mathrm{eV}$ ). The sensitivity to physical aging can be observed in isothermal conditions, but it appears clearly on the isochronal plots by comparison of the behavior exhibited on heating up to or above $T \mathrm{~g}$ and results obtained on cooling.

The fragile behavior is now to be explained from a microscopic point of view. Since the spacer is short we can expect a strong coupling between the motions of the chain segments and the mesogenic groups. As recalled in ref. [1], it is possible to take into account the correlated atomic motions in several ways. In the frame of the coupling model [5] the dynamics of relaxation proceeds at short time according a simple exponential. On elapsed time the relaxation rate is slowed down due to nonlinear interaction. The relaxation rate takes the form of a stretched exponential $\exp \left(-\left(t / \tau^{*}\right)^{1-n}\right)$. $\tau^{*}$ is identified to the mechanical relaxation time $\tau_{\text {mm }}$, while $n=1-b$ is the coupling parameter ( $n$ is equal to zero when molecular events proceed with no correlated effects). As a result, the apparent activation energy $E$ a observed in the isostructural state is: $E \mathrm{a}=E / b$, where $E$ is the energy barrier for the primary molecular event. From figure 3 $E \mathrm{a}$ is about $1.5 \mathrm{eV}$, which gives $E$ about $0.3 \mathrm{eV}$ which is not far from the activation energy of sub $T \mathrm{~g}$ relaxation processes [1]. This description, however, does not predict a value of $b$ ' lower than unity. From a microscopic point of view correlation of degrees of freedom over large distance are responsible for a low value of $b^{\prime}$. This remark is consistent with a decrease of $b^{\prime}$ as $x$ (i.e. crosslinking ratio) increases.

It is possible to deduce from the experiments complementary informations about the microstructure, namely the effect of crosslinking on the dynamics of relaxation. Examination of figure 2 indicates that in the glass transition region crosslinking makes motions easier on short range ( $\tan \varphi$ is higher at low temperature) and more difficult on long range $(\tan \varphi$ is lower at high temperature). It is deduced that after crosslinking an appreciable amount of degrees of freedom is present, which can be assigned to a local desorder or to residual monomers. Further investigation is necessary to clarify this point.

\section{References}

[1] Etienne S., David L., Mitov M., P. Sixou and Ngai K.L., Macromolecules 28 (1995) 5758-5764

[2] Etienne S., "Low frequency: Special Techniques", in Mechanical Spectroscopy in Materials Science, Magalas L.B. Ed., (Chapman and Hall, London, in press)

[3] Buijs J.A.H.M. and Vroege G.J., Polymer, 34 (1993) 4692-4696

[4] Angell C.A. In Relaxations in Complex Systems; Ngai K.L., Wright G.B., Eds.; (U.S. Government Printing Office: Washington DC, 1994) p3.

[5] Ngai K.L. In Disorder Effects on Relaxational Processes; Richert R., Blumen A., Eds; (Springer Verlag, New-York, 1994) pp89-150. 